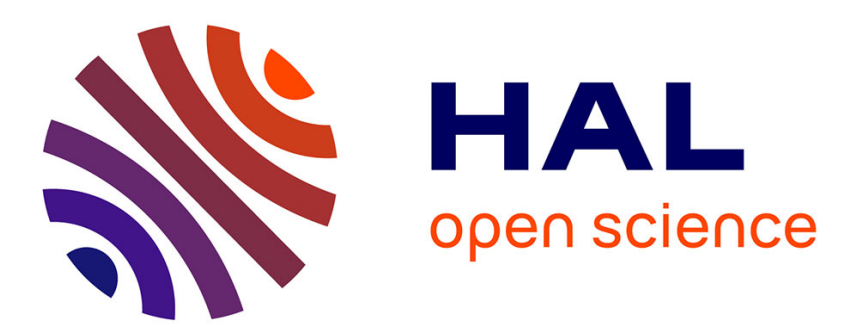

\title{
Internally Excited Fabry-Perot Type Cavity: Power Normalization and Directivity Evaluation
}

\author{
Halim Boutayeb, Tayeb Denidni
}

\section{To cite this version:}

Halim Boutayeb, Tayeb Denidni. Internally Excited Fabry-Perot Type Cavity: Power Normalization and Directivity Evaluation. IEEE Antennas and Wireless Propagation Letters, 2006, 5 (1), pp. 159162. 10.1109/LAWP.2006.873944 . hal-00149264

\section{HAL Id: hal-00149264 \\ https://hal.science/hal-00149264}

Submitted on 25 May 2007

HAL is a multi-disciplinary open access archive for the deposit and dissemination of scientific research documents, whether they are published or not. The documents may come from teaching and research institutions in France or abroad, or from public or private research centers.
L'archive ouverte pluridisciplinaire HAL, est destinée au dépôt et à la diffusion de documents scientifiques de niveau recherche, publiés ou non, émanant des établissements d'enseignement et de recherche français ou étrangers, des laboratoires publics ou privés. 


\title{
Internally-Excited Fabry-Perot Type Cavity: Power Normalization and Directivity Evaluation
}

\author{
Halim Boutayeb, Member, IEEE, and Tayeb A. Denidni, Senior Member, IEEE,
}

\begin{abstract}
This letter presents a new approach to analyze Fabry-Perot cavities excited from their inside by electromagnetic waves. While Fabry-Perot cavity antennas found in literature use often the magnitude of the transmission coefficient to determine the antenna directivity, the proposed approach suggests a new strategy that uses a normalized transmission coefficient, which is derived by using a transmission line model and by considering the available power from the source. Furthermore, a new analytical expression is also proposed for evaluating the directivity. The obtained results are presented and compared to experimental results reported in literature.
\end{abstract}

Index Terms - Fabry-Perot cavity, directive antennas.

\section{INTRODUCTION}

S EVERAL techniques for designing highly-directive antennas by incorporating a Fabry-Perot cavity have been proposed [1-8]. In [1], a relation between the half-power beamwidth and the quality factor of the cavity (at the resonant frequency of the cavity) has been proposed. In [2], the authors have demonstrated that this directivity improvement has an analogy with past research in optical physics. Another antenna consisting on a Fabry-Perot cavity between a patch antenna and an Electromagnetic Band Gap (EBG) material has been analyzed [3]. In addition, a Fabry-Perot cavity consisting on a Partially Reflecting Surface (PRS) and a ground plane has been used [4-7]. Furthermore, analytical expressions have been proposed in [8] for the frequency of maximum directivity and the minimum of half-power beamwidth of antennas based on a Fabry-Perot.

In the same perspective, a ray theory has been used by many authors for considering the problem of a source located inside a Fabry-Perot cavity [4-9]. This model is equivalent to the classical model of multiple wave reflections used for the Fabry-Perot interferometer in optics [10]. However, the source is considered inside the cavity instead of outside. For this case, the magnitude of the obtained transmission coefficient may achieve some values that can be greater than one. In [6, 7], this magnitude as been used for evaluating the antenna directivity.

To achieve a more accurate characterization for Fabry-Perot type cavities excited from their inside by electromagnetic waves, a new approach is proposed. This approach is based on a new normalized coefficient using a transmission line model and a new analytical expression for the evaluation of the

Manuscript received November 2005. This work was supported in part by the National Science Engineering Research Council of Canada (NSERC).

The authors are with Institut National de Recherche Scientifique (INRS)EMT, Montréal, Canada. Email : boutayeb@emt.inrs,ca, denidni@emt.inrs,ca antenna directivity. To validate this work, the obtained results are compared to experimental results reported in literature.

\section{RAY ANALYSIS}

For this study, a Fabry-Perot type cavity is considered. Fig. 1(a) shows this cavity. It is constituted by two Partially Reflecting Surfaces (PRSs) spaced by the distance $D$ and characterized by their complex transmission and reflection coefficients $t$ and $r$, for a plane wave incidence. For convenience, in this work, these coefficients are considered independent of frequency and incidence angle, which does not limit the studied problem. As illustrated in Fig. 1(a), a point source is considered inside the Fabry-Perot cavity. The source is considered transparent to electromagnetic waves and the characterization of the structure is obtained by using a one-dimensional model [8].

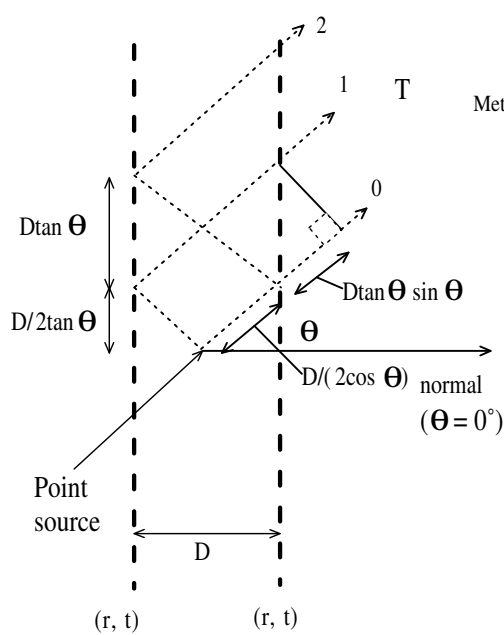

(a)

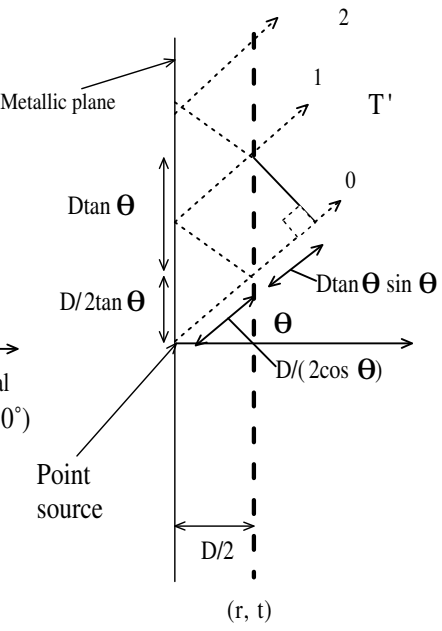

(b)
Fig. 1. (a) Fabry-Perot type cavity excited from its inside by a point source (in the center). (b) Fabry-Perot cavity constituted by a Partially Reflecting Surface and a metallic plane excited from its inside by a point source.

For a given angle $\theta$, considering the multiple wave reflections (see Fig. 1(a)), the amplitude of the transmitted wave outside the cavity is expressed as following [8]:

$$
\begin{aligned}
T(\theta, f) & =t \sum_{n=0}^{\infty} r^{n} e^{j k(2 n+1) D / 2 \tan \theta \sin \theta-\frac{j k(2 n+1) D}{2 \cos \theta}} \\
& =\frac{t e^{-j k D / 2 \cos \theta}}{1-r e^{-j k D \cos \theta}}
\end{aligned}
$$


where $k$ is the free space wave number. Note that a similar form is obtained if a ground plane is placed in the same position as the source [4-7], as illustrated in Fig 1(b) (there is just an additional $j \pi$ coefficient in the exponential of the denominator). For these two problems, illustrated in Fig. 1(a) and Fig. 1(b), the maximum transmitted power, achieved at resonance, is expressed as following [8]

$$
|T|_{\max }^{2}=\frac{1+|r|}{1-|r|}
$$

From Eq. (2), $|T|_{\max }^{2}$ is equal or greater than one and can theoretically become very large if $|r|$ is near one (note that $0 \leq|r| \leq 1$ ). The transmission coefficient greater than 1 means that the source inside the cavity can supply more power in the presence of the cavity than without the cavity.

In $[6,7]$, this relation has been used for evaluating the directivity of Fabry-Perot cavity based high-gain antenna.

\section{TRANSMISSION LINE MODEL : POWER NORMALIZATION}

According to the energy conservation principle, the transmission coefficient should not be greater than one. For this raison, a normalized version for the transmission coefficient is proposed taking into account the available power from the source. Note that, according the ray analysis, the response of the cavity of width $D$ at the angle $\theta$, is equivalent to response of the cavity of width $D \cos \theta$ at normal incidence. From this and according to circuit theory [11], the FabryPerot cavity excited from its interior can be presented by the transmission line model illustrated in Fig. 2(b). In Fig. 2(a), the transmission line model of the source with its matched impedance is also presented in order to calculate the available power from the source. $Z_{c}$ is the free-space characteristic impedance $\left(Z_{c}=120 \pi\right) . Z$ is the equivalent circuit model of the PRS. $Z_{s}$ and $e_{s}$ are the equivalent impedance and equivalent voltage of the source, respectively.

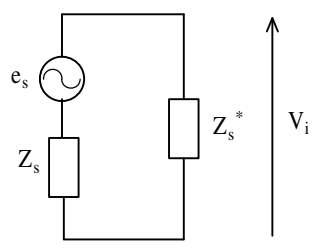

(a)

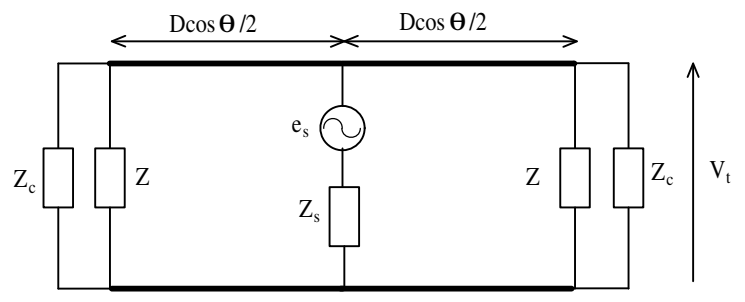

(b)

Fig. 2. Transmission line models: (a) Source with matched impedance (b) Source-Fabry Perot cavity structure.

To calculate the transmission coefficient outside the cavity, the available power $P_{i}$ (see Fig. 2(a)) is first calculated [11]:

$$
P_{i}=\frac{1}{2} \frac{\left|V_{i}\right|^{2}}{Z_{s}^{*}}=\frac{1}{2} \frac{\left|Z_{s}^{*}\right|^{2}}{Z_{s}^{*}\left|Z_{s}^{*}+Z_{s}\right|^{2}}\left|e_{s}\right|^{2}
$$

where $e_{s}, Z_{s}$ and $V_{i}$ are parameters shown in Fig. 2(a). Then referring to Fig. 2(b), the transmitted power $P_{t}$ for one side of the transmission line is given by:

$$
P_{t}=\frac{1}{2} \frac{\left|V_{t}\right|^{2}}{Z_{c}}
$$

To simplify the expressions, the transmission and reflection coefficients $t$ and $r$ can be expressed as functions of $Z$ [11]:

$$
\begin{gathered}
t=\frac{2 Z}{2 Z+Z_{c}} \\
r=-\frac{Z_{c}}{2 Z+Z_{c}}
\end{gathered}
$$

In addition, $t_{s}$ and $r_{s}$ are the transmission and reflection coefficients of the source, and they can be written :

$$
\begin{gathered}
t_{s}=\frac{2 Z_{s}}{2 Z_{s}+Z_{c}} \\
r_{s}=-\frac{Z_{c}}{2 Z_{s}+Z_{c}}
\end{gathered}
$$

In order to derive Eq. (4), Fig. 3 presents the equivalent model for the circuit of Fig. 2(b).

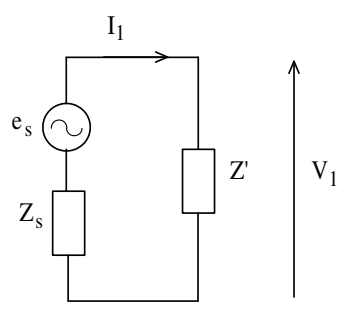

Fig. 3. Equivalent transmission line model of Fig. 2(b).

In this Figure, the impedance $Z^{\prime}$ can be written as following:

$$
\begin{aligned}
Z^{\prime} & =\frac{Z_{c}}{2} \frac{Z / / Z_{c}+j Z_{c} \tan (k \cos \theta D / 2)}{Z_{c}+j Z / / Z_{c} \tan (k \cos \theta D / 2)} \\
& =\frac{Z_{c}}{2} \frac{1-\frac{Z_{c}}{2 Z+Z_{c}} e^{-j k D \cos \theta}}{1+\frac{Z_{c}}{2 Z+Z_{c}} e^{-j k D \cos \theta}} \\
& =\frac{Z_{c}}{2} \frac{1+r e^{-j k D \cos \theta}}{1-r e^{-j k D \cos \theta}}
\end{aligned}
$$

The expression for the voltage $V_{1}$ (see Fig. 3) can be written:

$$
\begin{aligned}
V_{1} & =\frac{Z^{\prime}}{Z^{\prime}+Z_{s}} e_{s} \\
& =\frac{Z_{c}\left(1+r e^{-j k D \cos \theta}\right)}{\left(2 Z_{s}+Z_{c}\right)-\left(2 Z_{s}-Z_{c}\right) r e^{-j k D \cos \theta} e_{s}}
\end{aligned}
$$

And the current $I_{1}$ is expressed:

$$
I_{1}=\frac{V_{1}}{Z^{\prime}}=2 \frac{1-r e^{-j k D \cos \theta}}{\left(2 Z_{s}+Z_{c}\right)-\left(2 Z_{s}-Z_{c}\right) r e^{-j k D \cos \theta}} e_{s}
$$


Then, the voltage $V_{t}$ (Fig. 2(b)) is deduced from these results:

$$
\begin{aligned}
V_{t} & =V_{1} \cos (k \cos (\theta) D / 2)+j Z_{c} I_{1} / 2 \sin (k \cos (\theta) D / 2) \\
& =\frac{Z_{c}(1+r) e^{-j k D / 2 \cos \theta}}{\left(2 Z_{s}+Z_{c}\right)-\left(2 Z_{s}-Z_{c}\right) r e^{-j k D \cos \theta}} \\
& =\frac{Z_{c}}{2 Z_{s}+Z_{c}} \frac{(1+r) e^{-j k \cos \theta D / 2}}{1-\frac{2 Z_{s}-Z_{c}}{2 Z_{s}+Z_{c}} r e^{-j k D \cos \theta}} \\
& =\frac{Z_{c}}{2 Z_{s}+Z_{c}} \frac{t e^{-j k \cos \theta D / 2}}{1-\left(r_{s}+t_{s}\right) r e^{-j k D \cos \theta}}
\end{aligned}
$$

Thus, Eq. (4) can be written as

$$
P_{t}=\frac{1}{2} \frac{Z_{c}}{\left|2 Z_{s}+Z_{c}\right|^{2}} \frac{\left|t e^{-j k D \cos \theta / 2}\right|^{2}}{\left|1-r\left(r_{s}+t_{s}\right) e^{-j k D \cos \theta}\right|^{2}}\left|e_{s}\right|^{2}
$$

Taking into account the power transmitted from both sides of the line, the normalized transmitted power $|T|_{\text {Norm }}^{2}$ can be expressed as:

$$
\begin{aligned}
& |T|_{\text {Norm }}^{2}=\frac{2 P_{t}}{P_{i}} \\
& =\frac{2 Z_{c} Z_{s}^{*}\left|Z_{s}^{*}+Z_{s}\right|^{2}}{\left|Z_{s}^{*}\right|^{2}\left|2 Z_{s}+Z_{c}\right|^{2}} \frac{\left|t e^{-j k D \cos \theta / 2}\right|^{2}}{\left|1-r\left(r_{s}+t_{s}\right) e^{-j k D \cos \theta}\right|^{2}}
\end{aligned}
$$

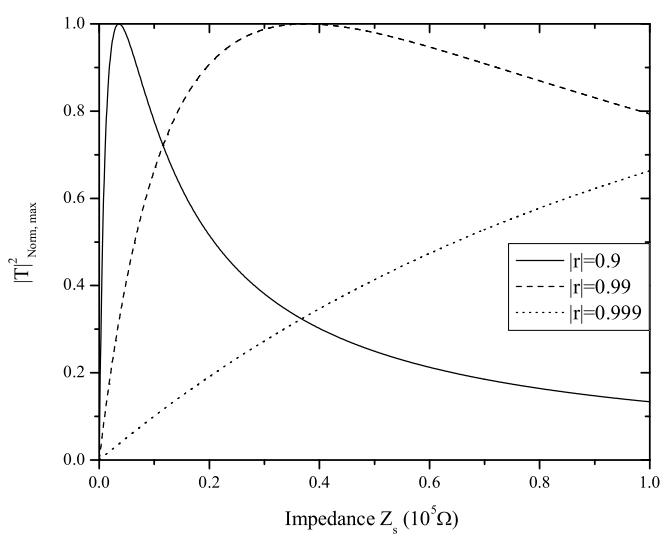

Fig. 4. $|T|_{\text {Norm, max }}^{2}$ coefficient versus $Z_{s}$ for different values of $|r|$.

For the case of real $Z_{s}$, Eq. (13) becomes

$$
|T|_{\text {Norm }}^{2}=4\left|r_{s}\right|\left|t_{s}\right| \frac{\left|t e^{-j k D \cos \theta / 2}\right|^{2}}{\left|1-r\left(r_{s}+t_{s}\right) e^{-j k D \cos \theta}\right|^{2}}
$$

The maximum is then written

$$
|T|_{\text {Norm,max }}^{2}=4\left|r_{s}\right|\left|t_{s}\right| \frac{1-|r|^{2}}{\left(1-|r|\left|r_{s}+t_{s}\right|\right)^{2}}
$$

In Fig. $4,|T|_{\text {Norm, } \max }^{2}$ is plotted versus $Z_{s}$ for different values of $|r|$. From these curves, one can see that the strength of $|T|_{\text {Norm }}^{2}$ is always limited to 1 .

\section{DISCUSSION}

Theoretically, Eq. (15) is more accurate than Eq. (1). However, Eq. (15) is less applicable in practice, because we don't have necessarily access to the transmission and reflection coefficients of the source. A rapid comparison between Eq. (1) and Eq. (15) shows that the normalized version (Eq. (15)) has two factors more: $4\left|r_{s}\right|\left|t_{s}\right|$ and $\left(r_{s}+t_{s}\right)$ (in the denominator). For instance, for directive-antennas incorporating a FabryPerot cavity, the angular dependence of the transmission coefficient is often used to evaluate the directivity. If the source is omnidirectional, the factor $4\left|r_{s}\right|\left|t_{s}\right|$ is independent on transmitting angle and it can then be omitted. Furthermore, the point source can be considered having a negligible interaction with the reflecting waves inside the cavity, which leads to $r_{s} \approx 0, t_{s} \approx 1$ and $r_{s}+t_{s} \approx 1$. From these, using Eq. (1) for predicting the radiation patterns and the directivity of directive-antennas based on a Fabry-Perot cavity excited internally is justified. However, the strength of the coefficient is not a direct evaluation of this directivity. The directivity is more associated with the inverse of the half power beamwidth. The half power beamwidth can be evaluated by using Eq. (1) as has been demonstrated in [8].

\section{DiRECTIVITY EVALUATION}

According to [8], the minimum of the half power beamwidth of an antenna based on a Faby-Perot cavity, $\Delta \theta_{3 d B, \text { min }}$, achieved near the resonant frequency, can be expressed as

$$
\Delta \theta_{3 d B, \min } \approx \sqrt{\frac{2}{Q}}
$$

where $Q$ is the quality factor and can be expressed as a function of the magnitude and phase of the reflection coefficient, $|r|$ and $\varphi_{r}$, as following

$$
\frac{1}{Q} \approx 2 \frac{1-|r|}{\phi_{r} \sqrt{|r|}}
$$

Using these relations and considering an antenna with an unique beam and same half-power beamwitdh in the H-plane and E-plane, the maximum of directivity of this antenna, is obtained approximatively with the following expression [12]

$$
D I R_{\max , d B} \approx 10 \log \frac{26000}{\left(\Delta \theta_{3 d B, \min } 180 / \pi\right)^{2}}
$$

The results obtained with this relation are compared to available experimental results in literature. In [6, 7], the values of the magnitude and phase of the reflection coefficient of the Partially Reflecting Surface (PRS), $|r|$ and $\varphi_{r}$ can be extracted. Using these values and Eq. (17)-(19), we obtain the maximum directivity of the proposed antenna. These results are compared to the measured maximum gain presented in the same references. Tab. 1 compares the directivity obtained analytically to the measured gain. From these results, one can see that the proposed analytical model gives accurate results.

Tab. 1: Comparaison between predicted results (Eq. (18)) and measured gain $[6,7]$.

\begin{tabular}{|c|c|c|c|}
\hline Reference & $|r|, \varphi_{r}(\mathrm{rad})$ & DIR $(\mathrm{dB})$ & Measured gain \\
\hline$[6]$ & $0.944,2.8798$ & 19.9 & 19 \\
\hline$[7]$ & $0.9788,2.9446$ & 24.34 & 21.9 \\
\hline
\end{tabular}




\section{CONCLUSION}

In this paper, a theoretical analysis on internally-excited Fabry-Perot cavity for directive antenna application, based on a one-dimensional model, has been presented. A new normalized coefficient using a transmission line model has been proposed. Then, a new analytical expression for the evaluation of the directivity of this type of antenna has also been formulated. The obtained results with the proposed analytical expression have been compared to experimental results available in literature. An excellent agreement was obtained between these results, demonstrating the accuracy and usefulness of our approach.

\section{REFERENCES}

[1] T. Akalin, J. Danglot, O. Vanbesien, and D. Lippens, "A Highly Directive Dipole Antenna Embedded in a Fabry-Perot Type Cavity", IEEE Microwave Wireless Comp. Lett., vol. 12, pp. 48-50, Feb. 2002.

[2] R. Biswas, E. Ozbay, B. Temelkuran, M. Bayandir, M. Sigalas, and K.-M. Ho, "Exceptionally directional sources with Photonic Band-Gap crystals", Optical Society of America, vol. 18, n. 11, pp. 1684-1689, Nov. 2001.

[3] M. Thevenot, C. Cheype, A. Reineix and B. Jecko, "Directive Photonic Band-Gap antennas", IEEE Trans. Microw. Theory Tech., vol. 47, pp. 21152122, Nov. 1999.

[4] G.V. Trentini, "Partially reflecting sheet arrays", IRE Trans. Antennas Propag., vol. 4, pp. 666-671, Oct. 1956.

[5] J.R. James, S.J.A. Kinany, P.D. Peel, and G. Andrasic, "Leaky-wave multiple dichroic beamformers", Electron. Lett., vol.25, $12091211,1989$.

[6] A.P. Feresidis, G. Goussetis, S. Wang and J.C. Vardaxoglou, "Artificial magnetic conductor surfaces and their application to low-profile high-gain planar antennas", IEEE Trans. Antennas Propag., vol. 53, pp. 209-215, Jan. 2005.

[7] A.P. Feresidis and J.C. Vardaxoglou, "High gain planar antenna using optimized partially reflective surfaces", IEE Proc.-Microw. Antennas Propag., vol. 148, pp. 345-350, Dec. 2001.

[8] H. Boutayeb, K. Mahdjoubi, A.C. Tarot, and T. Denidni "Directivity of an antenna embedded inside a Fabry-Perot cavity : Analysis and Design", Microw. Opt. Technol. Lett., vol. 48, pp. 12-17, Jan. 2006.

[9] B. Temelkuran, E. Ozbay, J. P Kavanaugh, G. Tuttle, and K.M. Ho, "Resonant cavity enhanced detectors embedded in photonic crystal", Appl. Phys. lett., vol. 72, pp. 2376-2378, May 1998.

[10] E. Hecht, Optics, Addison Wesley, San Francisco, CA, 2002, 421-425.

[11] D. Pozar, Microwave Engineering, 2nd. ed., New York, Wiley, 1998.

[12] C. A. Balanis, Antenna Theory: Analysis and Design, second Ed., John Wiley and Sons, p. 46 and p. 600, 1997. 